\title{
A DEGRADAÇÃO DA ESTEPE GRAMÍNEO-LENHOSA NO PARANÁ POR CONTAMINAÇÃO BIOLÓGICA DE Pinus elliottii E P. taeda
}

Sílvia Renate Ziller ${ }^{1}$ Franklin Galvão ${ }^{2}$

\begin{abstract}
RESUMO
Este trabalho contempla conceitos básicos de contaminação biológica, exemplos históricos de introdução de espécies e impactos decorrentes. Esses conceitos são aplicados à situação da Estepe Gramíneo-Lenhosa na região leste do Paraná, entre 790 e 1070 metros s.n.m., envolvendo os municípios de Ponta Grossa, Palmeira, Balsa Nova e Campo Largo. A descrição dos processos de degradação da Estepe em função das invasões por Pinus elliottii Engelm. e P. taeda L. leva em conta a forma de uso e ocupação do solo na região, calcado em sistema agrícola tradicionais e pastoreio extensivo que se utilizam queimadas anuais como forma de preparo do solo. São feitas recomendações para manejo florestal para reduzir esses impactos, considerando fatores físicos como posição no relevo, direção dos ventos predominantes e formato de povoamentos.
\end{abstract}

Palavras-chave: contaminação biológica, conservação ambiental, campos gerais planálticos.

\section{ENVIRONMENTAL DEGRADATION OF A GRASSLAND ECOSYSTEM IN PARANA STATE WITH BIOLOGICAL INVASIONS OF PINUS ELLIOTTI AND P. TAEDA}

\begin{abstract}
This paper establishes concepts and basic aspects of biological invasions, some examples of introductions and impacts. These data are applied to the grasslands suffering pine invasions from forest plantations in the eastern region of Paraná state, in the South of Brazil, between 790 and 1070 meters above sea level. The description of degradation processes by Pinus elliottii Engelm. and $P$. taeda L. invasions is linked to forms of land use and occupation in the region, based on annual burning for traditional agriculture and extensive grazing systems. Recommendations cover necessary improvements in the management of forest plantations with exotic species, considering factors such as relief, wind direction and plantation design.
\end{abstract}

Keywords: biological contamination, environmental conservation, grasslands, pine invasions.

\section{INTRODUÇÃO}

Este trabalho foi desenvolvido com o objetivo de descrever o processo de degradação ambiental decorrente da dispersão natural de Pinus elliottii Engelm. e P. taeda L. na Estepe Gramíneo-Lenhosa (IBGE, 1992) numa área de aproximadamente 91.400 hectares em Ponta Grossa, Palmeira, Balsa Nova e Campo Largo, no leste do estado do Paraná. Essa área equivale a 0,05\% do bioma, que compreende cerca de $19.000 \mathrm{~km}^{2}$ em toda a região Sul. São estabelecidos conceitos de contaminação biológica por espécies exóticas florestais e avaliados os impactos ambientais decorrentes de forma vinculada a processos erosivos e à prática anual de queima. Construída essa base, são feitas recomendações de planejamento e de manejo para mitigar o problema da invasão por Pinus spp. e a conseqüiente perda de biodiversidade.

\footnotetext{
1 sziller@sul.com.br, Eng. Florestal, M.Sc., Dr., Instituto Hórus de Desenvolvimento e Conservação Ambiental, R. Dr. Manoel Pedro, 495/906 Cabral Curitiba - PR 80.035-030

2 fgalvao@floresta.ufpr.br, Eng. Florestal, M.Sc., Dr., Departamento de Ciências Florestais da UFPR.

Recebido para publicação: 08/2001

Aceito para publicação: 05/2002
} 
Neste trabalho são discutidos apenas os ambientes afetados pela contaminação biológica por Pinus spp., não incluindo as formações florestais.

\section{REVISÃO BIBLIOGRÁFICA}

\section{Conceitos}

Contaminação biológica, ou invasão biológica é o processo de introdução e adaptação de espécies que não fazem parte, naturalmente, de um dado ecossistema, mas que se naturalizam e passam a provocar mudanças em seu funcionamento (Ziller, 2000).

Espécies exóticas são aquelas que ocorrem numa área fora de seu limite natural historicamente conhecido, como resultado de dispersão acidental ou intencional por atividades humanas (INSTITUTO DE RECURSOS MUNDIAIS; UNIÃO MUNDIAL PARA A NATUREZA; PROGRAMA DAS NAÇÕES UNIDAS PARA O MEIO AMBIENTE, 1992).

Espécies invasoras são aquelas que, uma vez introduzidas a partir de outros ambientes, se adaptam e passam a reproduzirse a ponto de ocupar o espaço de espécies nativas e produzir alterações nos processos ecológicos naturais, tendendo a tornar-se dominantes após um período de tempo mais ou menos longo requerido para sua adaptação (Ziller, 2000).

Sítios de disseminação são áreas a partir de onde a dispersão de sementes é potencializada em função de sua posição no relevo e/ou da direção dos ventos predominantes (Ledgard e Langer, 1999).

\section{História e finalidades da introdução de espécies}

As primeiras translocações de espécies de uma região a outra do planeta visavam, basicamente, suprir necessidades agrícolas, florestais e outras de uso direto. Em épocas mais recentes, o propósito das introduções de espécies tem se voltado significativamente para fins ornamentais, sendo que o número dessas espécies que se tornou invasora com o passar do tempo é de quase a metade dos casos registrados (Binggeli, 2000).
Na África do Sul, $42 \%$ de 491 espécies computadas até 1986 foram introduzidas com fins ornamentais, seguindo o uso para barreiras, cobertura, cultivo agrícola, forragem e silvicultura. Quanto maior a flexibilidade de uso da espécie maior é, em geral, seu potencial de se tornar invasora (Wells et al., 1986).

A Nova Zelândia, por sua vez, conta com mais de 24 mil espécies introduzidas, mais de $70 \%$ para fins ornamentais (DEPARTMENT OF CONSERVATION, 1998; BRAITHWAITE; TIMMINS, 1999). No Havaí, as espécies exóticas representam 47\% da flora atual (Wagner; Herbst; Sohmer, 1990). Estima-se que $31 \%$ de toda a área dos parques nacionais dos EUA esteja tomada por espécies exóticas invasoras, o que corresponde a 3,5 milhões de hectares (Westbrooks, 1998).

Além das finalidades de cultivo alimentar e de ornamentação para a introdução de espécies exóticas, há outras como produção florestal, controle de erosão, experimentação científica, camuflagem de instalações militares e usos medicinais e religiosos. Ainda, há o desejo de povos imigrantes de recriar a paisagem de suas terras de origem e até mesmo a idéia de melhorar a estética da paisagem, como foi o caso de semeaduras aéreas de coníferas exóticas na Montanha da Mesa, pano de fundo da Cidade do Cabo, e na reserva Ernesto Tornquist, nos pampas argentinos. Ambos os locais apresentam hoje problemas sérios de invasões biológicas em função dessas iniciativas (Wells et al., 1986; Richardson; Higgins, 1998 e Binggeli, 2000).

A experimentação florestal também gerou descendências atualmente custosas para diversos países. Houve esforços consideráveis, desde a metade do século XIX, de distribuição de sementes de espécies da América do Norte, notavelmente do gênero Pinus, e de espécies australianas como Melaleuca, Acacia, Hakea e Eucalyptus. Muitos dos plantios experimentais foram simplesmente abandonados por motivos diversos, dando às espécies espaço e tempo para adaptação e dispersão (Wells et al., 1986; Shaughnessy, 1986 e Corbett, 1991). Os gêneros Pinus e Eucalyptus são sem dúvida os mais utilizados mundialmente para produção florestal na atualidade, sendo que o primeiro apresenta imenso potencial invasor, com inúmeros registros para várias espécies no hemisfério Sul (Richardson, 1999). 
Exemplos brasileiros são a invasão por Pinus elliottii e $P$. taeda em ambiente de Formações Pioneiras de Influência Marinha (restinga) no Ribeirão da Ilha e nas praias do Moçambique e da Armação, na ilha de Santa Catarina (Caruso, 1990). A Estepe GramíneoLenhosa do segundo planalto paranaense está repleta de focos de invasão (Ziller, 2000) e são muitos os exemplos ao longo de estradas e áreas degradadas no sul do país onde essas espécies já se tornaram parte comum da paisagem.

\section{Impactos decorrentes de invasões biológicas}

O principal impacto causado por espécies exóticas invasoras refere-se à sua capacidade de modificar processos sistêmicos naturais, de forma que o processo de invasões biológicas é atualmente considerada a segunda maior ameaça à biodiversidade mundial, seguindo de perto a destruição de hábitats e a exploração humana direta, além de tratar-se de um problema subestimado pela maior parte dos países (D’Antonio; Vitousek, 1992; Randall, 1996; Hughes, 1994; Iucn, 2000).

Dentre os impactos mais citados no meio científico estão as alterações em processos ecológicos essenciais como ciclagem de nutrientes, produtividade vegetal, cadeias tróficas, estrutura, dominância, distribuição e funções de espécies, distribuição de biomassa, densidade de espécies, porte da vegetação, índice de área foliar, queda de serapilheira, taxa de decomposição, processos evolutivos e relações entre polinizadores e plantas. Podem mudar a adequação do hábitat para espécies animais, alterar características físicas do ecossistema, desencadeando processos erosivos, sedimentação e mudanças no ciclo hidrológico, no regime de incêndios e no balanço energético, além de reduzir o valor econômico da terra e o valor estético da paisagem, comprometendo seu potencial turístico. Podem ainda produzir híbridos ao cruzar com espécies nativas e eliminar genótipos originais e ocupar o espaço de plantas nativas levando-as a diminuir em densidade e extensão geográfica, aumentando os riscos de extinção de populações e de espécies. Os efeitos agregados de invasões potencializadas por atividades antrópicas põem em risco esforços para a conservação da biodiversidade, a manutenção da produtividade de sistemas agrícolas, a funcionalidade de ecossistemas naturais e a saúde humana (Breytenbach, 1986; Versfeld; Van Wilgen, 1986; Rapoport, 1991; D'Antonio; Vitousek, 1992; Westbrooks, 1998; Ledgard; Langer, 1999; Richardson, 1999; Higgins; Richardson; Cowling; Trinder-Smith, 1999; Mack et al., 2000).

É fato que a grande maioria dos países do mundo carece tanto de registros como de medidas de prevenção e controle de espécies invasoras, requerendo coleta e organização de dados para retratar a situação atual e para estabelecer prognósticos sobre o problema. Este é o caso do Brasil (Ziller, 2000).

\section{MATERIAIS E MÉTODOS}

A fim de produzir um diagnóstico das invasões biológicas, foram distribuídos na região do estudo 65 pontos utilizando o método de avaliação ecológica rápida (Sobrevilla; Bath, 1992). A área de estudo limitou-se à ocorrência de formações sedimentares, principalmente areníticas, da Formação Furnas e do Subgrupo Itararé.

A distribuição dos pontos diagnósticos foi definida de modo a cobrir os diferentes ambientes existentes, com apoio de imagens de satélite Landsat TM com resolução de 30 metros, havendo-se obtido coordenadas UTM com GPS Garmin 45 XL, com erro aproximado de 30 metros, para cada um dos pontos. Este trabalho é resultante da tese de doutorado de Ziller, 2000.

A Estepe Gramíneo-Lenhosa foi divida em seis subformações, a saber: Estepe stricto sensu, ou campo seco e limpo (17 pontos); Estepe higrófila, ou campo úmido (3 pontos); Formações Pioneiras de Influência Fluvial, ou brejos e várzeas (7 pontos); Refúgios Vegetacionais Rupestres, caracterizando a vegetação especializada a afloramentos de arenito (5 pontos); Floresta Ombrófila Mista Montana, ou capões com araucária (13 pontos); e Floresta Ombrófila Mista Aluvial, ou florestas de galeria (12 pontos). Além dessas formações, foram cobertas áreas agrícolas (4 pontos), povoamentos florestais ( 2 pontos) e pastagens ( 2 pontos) (Ziller, 2000). 


\section{RESULTADOS E DISCUSSÃO}

Composto basicamente de espécies heliófilas, o gênero Pinus tem sido registrado como potencial invasor de áreas abertas, sejam degradadas ou naturalmente ocupadas por vegetação herbáceo-arbustiva. Está registrado como invasor de ecossistemas abertos na Nova Zelândia, Austrália, África do Sul, Argentina, Chile e Brasil, entre outros países (Ziller, 2000). O gênero não se caracterizam, portanto, como invasor de formações florestais.

Árvores do gênero foram registradas em $76 \%$ dos pontos diagnósticos na Estepe stricto sensu, em 57\% dos pontos em Formações Pioneiras de Influência Fluvial e em $25 \%$ das áreas agrícolas. Muito embora as invasões sejam, na maior parte dos casos, incipientes, com árvores esparsas, é fundamental a noção de que esse problema aumenta gradativamente e se agrava com o passar do tempo, especialmente na total ausência de medidas de controle. É um processo inverso à maioria dos problemas ambientais, cujos impactos tendem a ser gradativamente absorvidos pelo meio. Isso leva à necessidade de aperfeiçoar o sistema tradicional de produção e manejo florestal de Pinus spp. e das outras atividades produtivas da região, visando mitigar os impactos decorrentes do uso de espécies exóticas invasoras.

Dentre as hipóteses que procuram explicar a suscetibilidade de ambientes à invasão biológica, a mais aceita e discutida é a que se fundamenta na ocorrência de perturbações no meio antes ou no momento em que ocorre a introdução de espécies exóticas. Assim sendo, é fundamental a noção das outras atividades produtivas que geram impactos ambientais na região (FIGURA 1), basicamente agricultura tradicional de milho, soja, feijão, trigo, aveia e azevém e criação de gado em sistema extensivo, na maior parte utilizando a vegetação nativa da Estepe como pasto. Os povoamentos florestais com Pinus spp. representavam, em 1998, cerca de $8 \%$ da área estudada, contra 64\% de Estepe stricto sensu, Estepe higrófila e Formações Pioneiras de Influência Fluvial, que são as formações mais suscetíveis à invasão por Pinus elliottii e $P$. taeda. A área agrícola correspondia a $16 \%$ e os restantes $12 \%$ representam a Floresta
Ombrófila Mista Montana e Aluvial e os talhões de Eucalyptus spp..

O preparo do solo para agricultura e a renovação do pasto para o gado são tradicionalmente feitos através de queimadas anuais, cuja periodicidade natural é estimada entre 8 e 15 anos. Esse processo leva a uma seleção gradativa de espécies mais resistentes ao fogo. De forma análoga, o freqüente sobrepastoreio leva à seleção de espécies de baixa palatabilidade ao gado, em geral de hábito arbustivo. $\mathrm{O}$ efeito primário de ambas as atividades é o desencadeamento de processos erosivos em função da exposição dos solos derivados de arenitos, levando em muitas áreas à involução pedológica dos Neossolos Litólicos da Estepe stricto sensu e dos Organossolos da Estepe higrófila, com posterior exposição da rocha subjacente.

$O$ efeito secundário é a mudança na ocorrência e na distribuição natural de espécies nativas da Estepe, com tendência à dominância de Baccharis spp. (carqueja) e Pteridium aquilinum (L.) Kuhn (samambaia-açu). Como conseqüência final tem-se, sob o aspecto econômico, a necessidade de aumento de área de pastagem natural por cabeça de gado e, sob o ponto de vista da conservação ambiental, a perda de biodiversidade.

Esses processos de degradação são altamente favoráveis à invasão e dispersão das árvores do gênero Pinus, pois tanto a queima como a erosão, expondo o solo e atingindo a bordadura dos capões e as florestas de galeria (FIGURA 1), dão às invasoras vantagens competitivas. A fotografia retrata a situação do Parque Estadual de Vila Velha em 1998. As árvores têm também a vantagem de serem resistentes ao fogo e de não serem palatáveis ao gado, portanto resistindo com facilidade a ambas as influências. A remoção de florestas ciliares para ampliação das áreas produtivas, aliada à alocação de povoamentos florestais às margens de cursos d'água, potencializa a dispersão de sementes e a instalação das invasoras ao longo dos mesmos. O mesmo se dá pela implantação de povoamentos nas porções mais elevadas do relevo regional, que, como as margens de cursos d'água, passam a constituir sítios de disseminação, principalmente por estarem expostos à ação dos ventos mais fortes, de direção predominante de nordeste. 

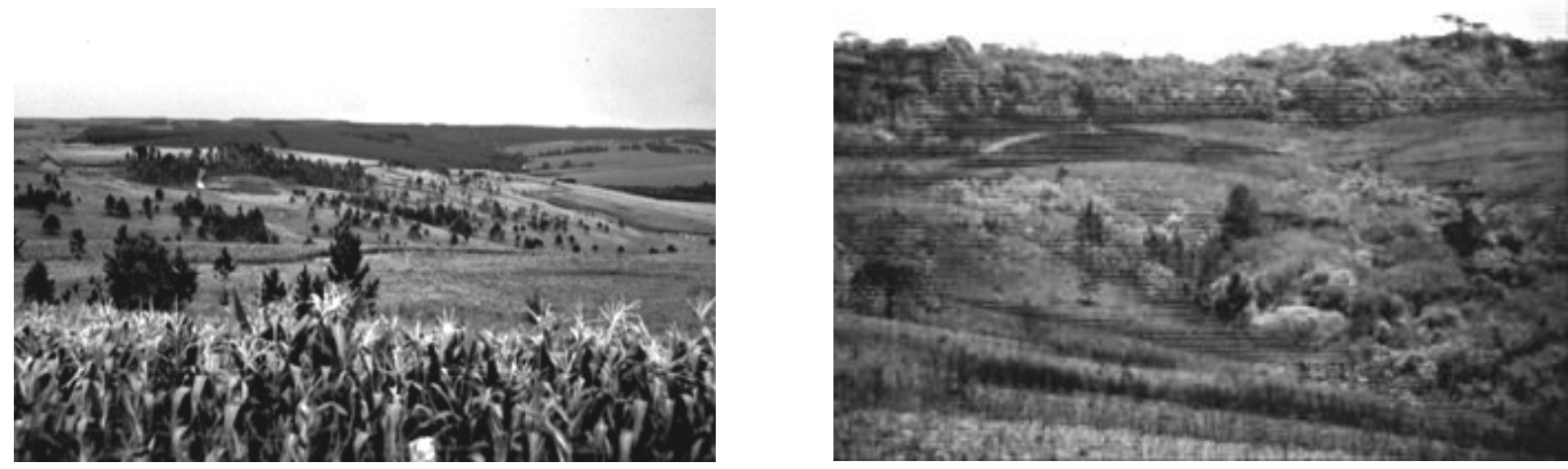

Figura 1: Agricultura, pasto e Pinus spp. invadindo os campos gerais no Paraná (esquerda) e Estepe e floresta após queima, com sobrevivência de Pinus spp. no Parque de Vila Velha (direita) (fotos: Sílvia Ziller, 1998).

Figure 1: Agriculture, pasture and Pinus spp. invading the general fields in Paraná (left) and Steppe and forest after it burns, with survival of Pinus spp. in the Park of Vila Velha (right) (photos: Sílvia Ziller, 1998).

\section{CONCLUSÕES E RECOMENDAÇÕES}

É fundamental ressaltar que não são as atividades econômicas em si as responsáveis pela degradação ambiental geral da Estepe Gramíneo-Lenhosa, mas sim a forma como são desenvolvidas. O resultado implica perda de biodiversidade de um ecossistema sobre o qual não há informação científica suficiente para levar a ações satisfatórias de restauração ambiental, tomando por princípio que as mesmas devem buscar a restituição de processos ecológicos naturais. $\mathrm{O}$ uso tradicional do meio tende a inviabilizar a sustentabilidade dos recursos naturais na região, de forma que são a seguir propostas algumas alternativas para mitigar os atuais impactos decorrentes dessas atividades. Essas recomendações estão fundamentadas na extensão do trabalho de doutoramento e podem ser buscadas em maior detalhe em Ziller, 2000.

- Substituir o sistema de pastoreio extensivo pelo manejo rotativo racional (VOISIN, 1974), visando viabilizar a recuperação do pasto nativo entre períodos de pastejo. Esse sistema evita a seleção de espécies não palatáveis ao gado e prescinde da necessidade de queimadas para regenerar a vegetação nativa da Estepe;
- definir o formato dos povoamentos florestais de acordo com características locais de relevo, posição de cursos d'água e direção dos ventos, conforme situação específica local, visando maximizar a dispersão de sementes de Pinus spp. dentro dos próprios povoamentos (veja representação na FIGURA 2);

- estabelecer ao redor dos povoamentos florestais três faixas de quebra-vento com espécies não invasoras a fim de reduzir a dispersão de sementes;

- preferencializar o estabelecimento de povoamentos florestais em meias encostas, a fim de evitar a formação de sítios de disseminação em topos e vales;

- manter e/ou restaurar florestas de galeria como medida de proteção à disseminação de sementes por cursos d'água;

- estabelecer processos de certificação ambiental para a região, previstos a prevenção, o controle e o monitoramento da dispersão natural de plantas invasoras;

- restringir o uso do gênero Pinus à produção florestal na região a fim de reduzir as áreas-fonte de contaminação biológica do bioma dos campos sulinos. 


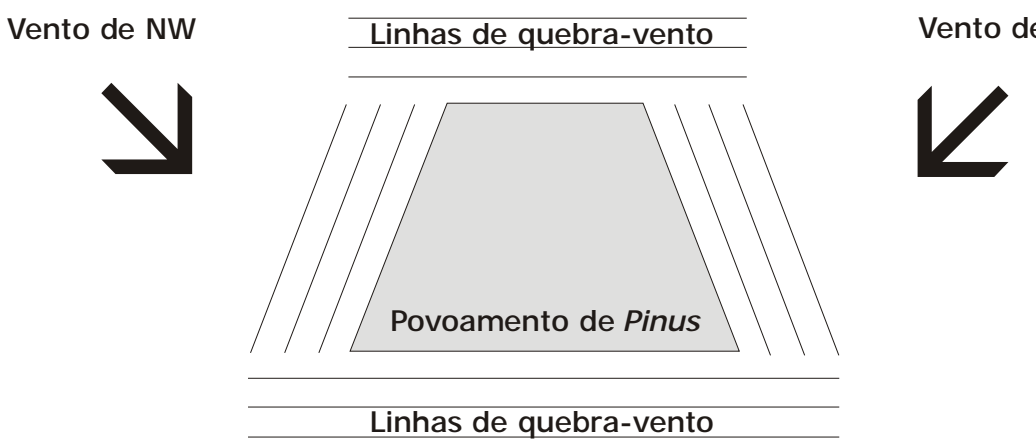

Figura 2: Povoamento florestal em forma de trapézio, definida em função da direção dos ventos predominantes no verão $(\mathrm{NE})$ e inverno $(\mathrm{NW})$. Visa manter a grande parte da dispersão de sementes no seu próprio interior, o que também é gerado pelas linhas de quebra-vento ao longo de todas as laterais (ZILLER, 2000).

Figure 2: Forest stand design defined according to dominant winds, intending to keep most seed dispersal inside. Shelter belts also minimize dispersal

\section{BIBLIOGRAFIA CITADA}

BINGGELI, P. The human dimensions of invasive woody plants. No prelo. http://members.tripod.co.uk/WoodyPlantEcolo gy. 2000.

BRAITHWAITE, H.; TIMMINS, S.M. Weed surveillance - catching 'em early. In: $\mathbf{1 2}^{\text {th }}$ Australian Weed Conference, Hobart, Tasmania, Austrália, Sep. 1999.

BREYTENBACH, G.J. Impacts of alien organisms on terrestrial communities with emphasis on communities of the southwestern Cape. In: MACDONALD, I.A.W.; KRUGER, F.J.; FERRAR, A.A. The ecology and management of biological invasions in Southern Africa. Cape Town: Oxford University Press. p. 229-238. 1986.

CORBETT, D.P. Control of cluster pine on French Island, Victoria. Plant Protection Quarterly, Austrália, v. 6, n. 3, 128 p. 1991.

D'ANTONIO, C.M.; VITOUSEK, P.M. Biological invasions by exotic grasses, the grass/fire cycle, and global change. Annual Rev. Ecol. Syst., n. 23, p. 63-87. 1992.

DEPARTMENT OF CONSERVATION. Space invaders - a summary of the Department of Conservation's Strategic Plan for Managing Invasive Weeds. Wellington: The Science Publications Unit,
Department of Conservation, October. 28 p. 1998.

HIGGINS, S.I.; RICHARDSON, D.M.; COWLING, R.M.; TRINDER-SMITH, T.H. Predicting the landscape-scale distribution of alien plants and their threat to plant diversity. Conservation Biology, v. 13, n. 2, p. 303-313, Apr. 1999.

HUGHES, C.E. Risks of species introductions in tropical forestry. Commonwealth Forestry Review, v. 73, n. 4, p. 243-252. 1994.

INSTITUTO DE RECURSOS MUNDIAIS; UNIÃO MUNDIAL PARA A NATUREZA; PROGRAMA DAS NAÇÕES UNIDAS PARA O MEIO AMBIENTE. A estratégia global da biodiversidade - diretrizes de ação para estudar, salvar e usar de maneira sustentável e justa a riqueza biótica da Terra. Curitiba: World Resources Institute / Fundação O Boticário de Proteção à Natureza. 232 p. 1992.

INSTITUTO BRASILEIRO DE GEOGRAFIA E ESTATÍSTICA (IBGE). Manual de classificação da vegetação brasileira. Série Manuais Técnicos em Geociências, $n^{\circ} 1$. Rio de Janeiro: IBGE, 92 p. 1992.

IUCN - INTERNATIONAL UNION FOR CONSERVATION OF NATURE AND NATURAL RESOURCES. IUCN guidelines 
for the prevention of biodiversity loss caused by alien invasive species. 51st meeting of Council, February. 2000.

LEDGARD, N.J.; LANGER, E.R. Wilding prevention - guidelines for minimising the risk of unwanted wilding spread from new plantings of introduced conifers. New Zealand: Forest Research. 20 p. 1999.

MAACK, R. Geografia física do estado do Paraná. 2 ed. Rio de Janeiro: Livraria José Olympio Editora, 442 p. 1981.

MACK, R.N.; CHAIR; SIMBERLOFF, D.; LONSDALE, W.M.; EVANS, H.; CLOUT, M.; BAZZAZ, F. Biotic invasions: causes, epidemiology, global consequences and control. In: Issues in Ecology n. 5, Spring. 20p. 2000.

RANDALL, J.M. Weed control for the preservation of biological diversity. Weed technology: n. 10, p. 370-383. 1996.

RAPOPORT, E.H. Contaminação por espécies. Ciência Hoje, v. 13, n. 75, p. 52-57, ago. 1991.

RICHARDSON, D.M. Commercial forestry and agroforestry as sources of invasive alien trees and shrubs. In: SANDLUND, O.T.; SCHEI, P.J.; VIKEN, A. Invasive species and biodiversity management. Dordrecht: Kluwer Academic Publishers. p. 237-257. 1999.

SHAUGHNESSY, G. A case study of some woody plant introductions to the Cape Town area. In: MACDONALD, I.A.W.; KRUGER, F.J.; FERRARA, A.A. The ecology and management of biological invasions in southern Africa. Cape Town: Oxford University Press. p. 37-43. 1986.

SOBREVILLA, C.; BATH, P. Evaluacion ecologica rapida - un manual para usuarios de América Latina y el Caribe. Rel. técnico. Washington: The Nature Conservancy, 232 p. feb. 1992.

VERSFELD, D.B.; VAN WILGEN, B.W. Impact of woody aliens on ecosystem properties. in: MACDONALD, I.A.W.; KRUGER, F.J.; FERRARA, A.A. The ecology and management of biological invasions in southern Africa. Cape Town: Oxford University Press. p. 239-246. 1986.
WAGNER, W.L., HERBST, D.R.; SOHMER, S.H. Manual of the flowering plants of Hawaii. 2v. Bishop Museum Special Publication 83. University of Hawaii Press and Bishop Museum Press, Honolulu, Hawaii. 1854 p. 1990.

WELLS, M.J.; POYNTON, R.J.; BALSINHAS, A.A.; MUSIL, K.J.; JOFFE, H.; VAN HOEPEN, E.; ABBOTT, S.K. The history of introduction of invasive alien plants to southern Africa. In: MACDONALD, I.A.W.; KRUGER, F.J.; FERRARA, A.A. The ecology and management of biological invasions in southern Africa. Cape Town: Oxford University Press. p. 21-35. 1986.

WESTBROOKS, R. Invasive plants: changing the landscape of America: fact book. Washington, DC: Federal Interagency Committee for the Management of Noxious and Exotic Weeds. 107 p. 1998.

ZILLER, S.R. A Estepe Gramíneo-Lenhosa no segundo planalto do Paraná: diagnóstico ambiental com enfoque à contaminação biológica. Tese de doutoramento. Curitiba: Universidade Federal do Paraná. 268 p. 2000. 